\title{
Concomitância de fibromialgia em pacientes com espondilite anquilosante
}

\author{
Valderilio Feijó Azevedo ${ }^{1}$ Eduardo dos Santos Paiva ${ }^{1}$, Lúcio Ricardo Hiurko Felippe ${ }^{2}$, Ranieri Amorim Moreira ${ }^{3}$
}

\begin{abstract}
RESUMO
Introdução: A Espondilite Anquilosante (EA) é uma doença inflamatória crônica que acomete o esqueleto axial. Cursa com dor e incapacidade funcional. Para medir o impacto da EA na vida dos pacientes são utilizados questionários que avaliam a atividade da doença (BASDAI); a incapacidade funcional (BASFI); e a qualidade de vida (ASQoL). A Fibromialgia (FM) é uma das causas mais comuns de dor generalizada e pode coexistir com outras doenças; pode ser avaliada por meio do questionário de impacto da Fibromialgia (FIQ). Há poucos estudos demonstrando correlações entre FM e EA. O presente estudo obteve dados referentes ao perfil epidemiológico de pacientes com EA e FM e avaliou a prevalência de FM em portadores de EA. Avaliou-se a interferência da FM nos escores dos testes BASDAI, BASFI e ASQoL. Pacientes e Método: Foram incluídos 71 pacientes portadores de EA diagnosticados de acordo com os critérios modificados de Nova York. Avaliação clínica, funcional e aplicação dos testes BASDAI, BASFI e ASQoL foram realizados. Os pacientes com diagnóstico de FM foram avaliados com o FIQ. Resultados: Onze pacientes preencheram os critérios para FM, observando-se assim uma prevalência de 15\% de FM entre os pacientes com EA, sendo mais frequente entre as mulheres (3,8:1). A idade de início da doença (EA) foi de 27,5 anos. O antígeno HLA-B27 foi positivo na grande maioria $(80,4 \%)$. Comparando-se as médias dos testes BASDAI, BASFI e ASQoL, verificou-se que os valores são significativamente superiores $(\mathrm{P}<0,01)$ entre os pacientes com FM. Concluiu-se que a coexistência da FM pode piorar os aspectos de atividade na EA, a incapacidade funcional e a qualidade de vida.
\end{abstract}

Palavras-chave: espondilite anquilosante, fibromialgia, avaliação do perfil de impacto da doença.

\section{INTRODUÇÃO}

A espondilite anquilosante (EA) é uma doença inflamatória crônica que acomete preferencialmente a coluna vertebral, podendo evoluir com rigidez e limitação funcional progressiva do esqueleto axial. ${ }^{1,2}$ Mais frequente em adultos jovens, com idade de início geralmente entre 20 e 40 anos, tem prevalência maior no sexo masculino (3:1), caucasianos e em indivíduos HLA-B27 positivos. ${ }^{2,3}$ Faz parte do complexo das espondiloartrites no qual o HLA-B27 está fortemente correlacionado, com sua presença variando entre $80 \%$ e $98 \%$ dos casos. ${ }^{4}$

O quadro clínico apresenta inicialmente lombalgia de ritmo inflamatório associada a rigidez matinal. ${ }^{1,2} \mathrm{Na}$ coluna, a EA promove ossificações ligamentares, fusão de vértebras, osteoporose, resultando em uma coluna vertebral enfraquecida, remodelada, com forte tendência a fraturas e deformações. ${ }^{1,5}$ As entesopatias são caracterizadas por inflamações nas inserções dos tendões e/ou ligamentos nos ossos e acometem preferencialmente a inserção do tendão do calcâneo e a fáscia plantar. ${ }^{1}$

Para a confirmação diagnóstica da EA, os critérios mais utilizados são os de Nova York modificados, que combinam critérios clínicos e radiográficos. Para o diagnóstico de EA é necessária a presença de um critério clínico e um critério radiográfico. ${ }^{6}$

Nas últimas décadas, devido ao surgimento de medicações mais eficazes para o tratamento da EA e à necessidade de se

Recebido em 22/4/2010. Aprovado, após revisão, em 11/11/2010. Declaramos a inexistência de conflito de interesse.

Hospital de Clínicas da Universidade Federal do Praná - UFPR.

1. Professor Assistente em reumatologia, MD

2. Psicólogo aluno da graduação médica da UFPR - aluno da graduação médica UFPR

3. Estudante de medicina UFPR - aluno da graduação médica da UFPR

Endereço para correspondência: Valderilio Feijó Azevedo. Rua Lamenha Lins 1110, ap 11ª, Rebouças. Curitiba, PR, Brasil. CEP 80250-020.

E-mail: valderilio@hotmail.com. 
tornar objetiva e padronizada a avaliação clínica dos pacientes, diversos instrumentos foram propostos.

Em 1994, Garret et al. apresentaram um questionário a fim de avaliar a atividade da EA. ${ }^{7} \mathrm{O}$ teste BASDAI (Bath Ankylosing Spondylitis Disease Activity Index) é um questionário que consiste em seis questões que abordam domínios relacionados à fadiga, dor na coluna, dor e sintomas articulares, dor devido ao acometimento das enteses, e duas questões relacionadas à qualidade e quantidade de rigidez matinal. $\mathrm{O}$ escore é medido em escalas visuais analógicas (EVA) de 0 a 10 $(0=$ bom; $10=$ ruim $)$. Atualmente é considerado um dos mais importantes instrumentos para a utilização em ensaios clínicos. ${ }^{8}$

Em 1994, Calin et al. publicaram o BASFI (Bath Ankylosing Spondylitis Functional Index), questionário que objetiva a avaliação da limitação de funcionalidade presente nos pacientes de EA. ${ }^{9} \mathrm{O}$ teste inclui oito itens relacionados a atividades da vida diária e dois itens que medem as habilidades do paciente em lidar com o seu dia a dia. ${ }^{9}$

Ambos os critérios, BASFI e BASDAI, foram bem aceitos pela praticidade, funcionalidade, reprodutibilidade e sensibilidade para apreciar as características da EA e sua evolução. Muitos países já fizeram a validação ${ }^{10,11}$ desse método de acordo com sua língua, assim como o Brasil.

Pela importância da avaliação da qualidade de vida como medida de desfecho em estudos clínicos, questionários como o ASQoL (Ankylosing Spondylitis Quality of Life Questionnaire) têm sido cada vez mais utilizados. O teste engloba 18 questões com resposta sim ou não, resultando em um escore de 0 a 18 , com o maior valor implicando uma pior qualidade de vida. É o único desenvolvido originalmente para a medida específica de qualidade de vida em EA. ${ }^{8,12}$

A fibromialgia (FM) é uma das causas mais comuns de dor musculoesquelética generalizada. A etiologia permanece ainda desconhecida, mas acredita-se não se tratar de um processo inflamatório. É considerada uma síndrome de amplificação da dor, associada a um mecanismo de sensibilização do sistema nervoso central. ${ }^{13,14}$ Ocorre mais frequentemente em mulheres e a maioria dos pacientes está entre 35 e 50 anos. No exame físico é notória a ausência de sinovite e/ou outros sinais indicativos de doença inflamatória; a principal característica é a presença de áreas de hipersensibilidade (pontos dolorosos) identificadas à palpação..$^{14,15}$

A FM pode coexistir com outras doenças reumatológicas como artrite psoriásica $(24 \%),{ }^{16}$ artrite reumatóide $(25 \%),{ }^{13}$ lúpus eritematoso sistêmico (30\%) ${ }^{13}$ e doença de Crohn (49\%). ${ }^{17}$

O diagnóstico da FM é essencialmente clínico e baseia-se no achado de pontos dolorosos e na ausência de sintomas ou achados laboratoriais que indiquem uma doença inflamatória ou degenerativa. Nesse sentido, as provas de atividade inflamatória, as enzimas musculares e eletromiografia são normais..$^{13,15}$ Em pesquisa utilizam-se os critérios do Colégio Americano de Reumatologia: 1) dor generalizada crônica em ambos os lados do corpo, tanto axial quanto periférica, abaixo e acima da cintura; 2) presença de 11 de 18 pontos dolorosos. ${ }^{18}$

Há poucos estudos demonstrando correlações entre a FM e EA. Um único artigo foi publicado apresentando a prevalência da FM em pacientes com EA. Este mesmo artigo também apontou para a interferência que a coexistência da FM promove nos escores dos testes de avaliação do paciente. ${ }^{19}$

A proposta do presente estudo foi a de obtenção de dados referentes ao perfil epidemiológico de pacientes com EA e FM e identificação da prevalência de FM em portadores de EA. Também pretendeu comparar os índices funcionais e avaliou se a coexistência do quadro de FM pode interferir nos escores dos testes de avaliação da atividade da doença (BASDAI), avaliação funcional (BASFI) e qualidade de vida do paciente com EA (ASQLo).

\section{PACIENTES E MÉTODO}

\section{População-alvo}

O estudo avaliou 71 pacientes com EA diagnosticados de acordo com os critérios modificados de Nova Iorque, atendidos no Ambulatório de Reumatologia do Hospital de Clínicas de Curitiba, no período de abril a dezembro de 2009. Pacientes que apresentassem concomitantemente outra doença reumatológica que justificasse a presença de dor crônica generalizada foram excluídos. Todos os pacientes deram seu consentimento informado para participar deste estudo, que foi aprovado pelo Comitê de Ética em Pesquisas do Hospital sob número 1890.057/2009-03.

\section{Procedimentos}

Foi realizado um estudo epidemiológico observacional transversal de casos prevalentes. Os pacientes responderam a um questionário que incluiu as seguintes informações: data de nascimento, idade, sexo, cor da pele, data de início dos sintomas; foi pesquisada a história mórbida familiar quanto à presença de EA em parentes como irmãos e pais. Foram coletados os dados do prontuário médico referentes à pesquisa do antígeno HLA-B27. A avaliação da atividade da doença foi investigada por meio da aplicação do BASDAI, a avaliação funcional por meio do BASFI e a qualidade de vida do paciente com EA por meio do ASQoL. Na sequência os pacientes foram avaliados 
quanto à presença de FM segundo os critérios do Colégio Americano de Reumatologia, sendo considerados positivos quando presentes os dois critérios concomitantemente.

\section{Processamento e análise dos dados}

Os pacientes foram separados em 2 grupos: com e sem FM. A pontuação dos testes BASDAI, BASFI e ASQoL dos 2 grupos foi comparada por meio do teste $t$ de Student e teste Wilcoxon.

\section{RESULTADOS}

Os dados epidemiológicos dos 71 pacientes avaliados encontram-se na Tabela 1 . Destes $84,5 \%$ eram homens e $15,5 \%$ eram mulheres, (proporção de 5,5 homens para cada mulher). Dos 71 pacientes avaliados, $11(15 \%)$ preencheram os critérios

\section{Tabela 1}

Dados epidemiológicos e clínicos dos 71 participantes do estudo com diagnóstico de espondilite anquilosante

\begin{tabular}{lc}
\hline Características & Valores \\
\hline Total de pacientes & 71 \\
$\quad$ Homens & $54(84,5 \%)$ \\
$\quad$ Mulheres & $17(15,5 \%)$ \\
\hline Pacientes com Fibromialgia & $11(15 \%)$ \\
$\quad$ Homens & $5(45,5 \%)$ \\
$\quad$ Mulheres & $6(54,5 \%)$ \\
\hline Idade (anos) & $43,67(\mathrm{DP}=11,78)$ \\
\hline Idade de início dos sintomas (anos) & $27,5(\mathrm{DP}=11,80)$ \\
Duração dos sintomas (anos) & $16,59(\mathrm{DP}=9,64)$ \\
\hline Histórico Familiar & \\
\hline$\quad$ Positivo (\%) & 13,2 \\
\hline Negativo (\%) & 86,8 \\
\hline HLA-B27 & \\
Positivo (\%) & 80,4 \\
$\quad$ Negativo (\%) & 16,6 \\
\hline
\end{tabular}

DP: desvio-padrão. do Colégio Americano de Reumatologia para FM, sendo 5 $(45,5 \%)$ homens e $6(54,5 \%)$ mulheres. A prevalência da FM foi de $15 \%$ entre os pacientes com EA, e entre os sexos, a proporção foi de 3,8 mulheres para cada homem com $\operatorname{FM}(3,8: 1)$.

A idade média dos pacientes foi de 43,67 (desvio-padrão $[D P]=11,78)$, variando entre 19 e 69,3 anos. A idade média de início dos sintomas foi de 27,5 anos ( $\mathrm{DP}=11,80)$. A duração dos sintomas teve tempo médio de 16,59 anos $(\mathrm{DP}=9,64)$. A maioria dos pacientes tem cor de pele branca $(70,4 \%)$ ou parda $(21,1 \%)$. Apenas 13,2\% dos pacientes apresentaram histórico familiar positivo para espondiloartrites. Dos 71 pacientes avaliados, 46 (65\%) apresentavam informações sobre o antígeno HLA-B27 em seus prontuários, sendo positivo na grande maioria $(80,4 \%)$.

Os dados comparativos dos dois subgrupos encontram-se na Tabela 2 e Figura 1.

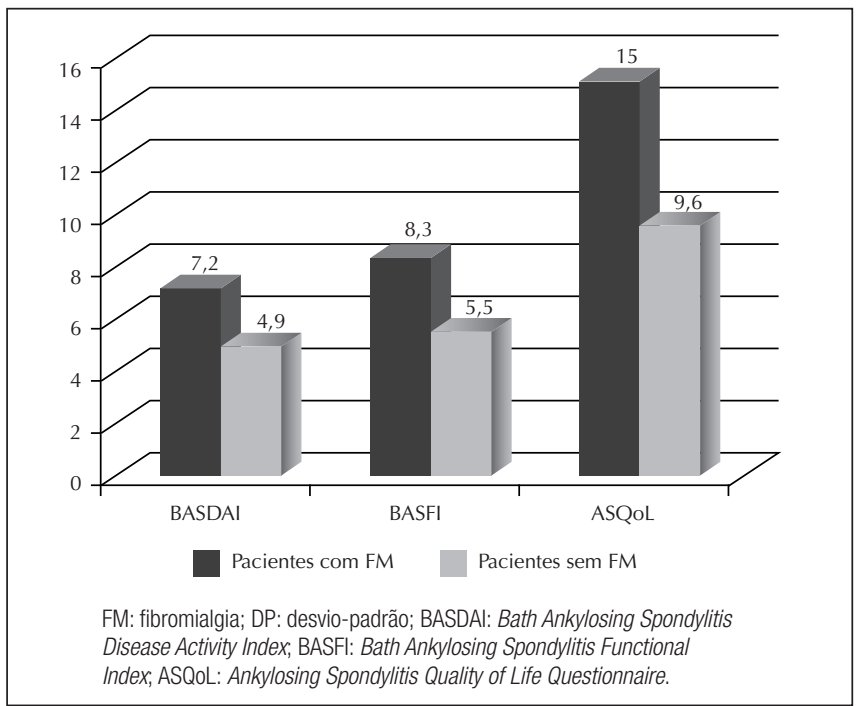

\section{Figura 1}

Comparação entre as médias dos escores dos testes BASDAI, BASFI e ASQoL dos 2 subgrupos com e sem FM. Em todos os testes houve diferença estatística significativa, confirmada com o teste $t$ de Student e teste Wilcoxon.

Tabela 2

Dados comparativos dos testes BASDAI, BASFI e ASQoL entre os pacientes com e sem FM

\begin{tabular}{lcccc}
\hline Testes & $\begin{array}{c}\text { Pacientes com FM } \\
\mathbf{n = 1 1}\end{array}$ & $\begin{array}{c}\text { Pacientes sem FM } \\
\mathbf{n = 6 0}\end{array}$ & $\begin{array}{c}\text { P-value } \\
\text { (teste } \boldsymbol{t} \text { de Student) }\end{array}$ & $\begin{array}{c}\text { P-value } \\
\text { (teste Wilcoxon) }\end{array}$ \\
Número de Pacientes & 11 & 60 & & \\
BASDAI & $7,2(\mathrm{DP}=1,61)$ & $4,9(\mathrm{DP}=2,38)$ & $<0,001$ & $=0,002940$ \\
BASFI & $8,3(\mathrm{DP}=1,03)$ & $5,5(\mathrm{DP}=3,11)$ & $<0,001$ & $=0,001992$ \\
ASQoL & $15(\mathrm{DP}=2,00)$ & $9,6(\mathrm{DP}=5,55)$ & $=0,0019$ & $=0,001884$ \\
\hline
\end{tabular}

FM: fibromialgia; DP: desvio-padrão; BASDAl: Bath Ankylosing Spondylitis Disease Activity Index

BASFI: Bath Ankylosing Spondylitis Functional Index, ASQoL: Ankylosing Spondylitis Quality of Life Questionnaire. 


\section{DISCUSSÃO}

Os dados epidemiológicos apresentados neste estudo corroboraram com os dados encontrados na literatura. A média de idade de início da doença dos nossos pacientes foi de 27,5 anos, dentro dos parâmetros de 20 a 40 anos, ou seja, início dos sintomas tipicamente na faixa etária de adultos jovens. ${ }^{4} \mathrm{~A}$ prevalência foi maior no sexo masculino $(5,5: 1)$ e um pouco acima dos dados da literatura $(3: 1){ }^{4}$ A doença se apresentou mais prevalente em indivíduos da cor branca $(70,4 \%$ dos pacientes pesquisados). $\mathrm{O}$ antígeno HLA-B27 foi positivo na grande maioria $(80,4 \%) .{ }^{20}$

Embora tenhamos encontrado baixo histórico familiar de espondiloartrites na maioria dos pacientes $(86,8 \%)$, os fatores genéticos classicamente contribuem para uma maior suscetibilidade para o desenvolvimento da doença. Verificou-se que o risco de desenvolver EA é muito maior (10 a 20 vezes mais) entre os parentes de pacientes com HLA-B27 positivo, comparado com a população em geral com HLA-B27 positivo. ${ }^{21}$

Um único estudo identificou a prevalência da FM entre pacientes com EA. ${ }^{19}$ Aloush et al. estudaram um grupo de 36 pacientes com diagnóstico de EA, sendo 18 homens e 18 mulheres. Nesse estudo confirmaram o diagnóstico de FM em $50 \%$ das mulheres, porém não confirmaram entre nenhum dos homens. Os pesquisadores observaram que os pacientes que apresentavam concomitância das duas doenças possuíam prejuízos funcionais mais graves, o que refletia escores aumentados nos testes BASDAI e BASFI, quando comparados aos escores dos pacientes portadores de uma dessas doenças isoladamente. Um viés importante estava presente e colocou em dúvida se a presença da FM poderia influenciar o resultado dos testes de avaliação funcional: o diagnóstico de FM foi encontrado apenas no grupo das mulheres. ${ }^{19}$ Sabe-se que a manifestação da EA é diferente entre os sexos, sendo de pior prognóstico entre homens. ${ }^{22}$

Em nosso estudo, 71 pacientes foram avaliados e a prevalência da FM foi de $15 \%$, sendo que neste grupo 45,5\% eram homens e $54,5 \%$ eram mulheres. Avaliamos uma amostra mais balanceada entre homens $(45,5 \%)$ e mulheres $(54,5 \%)$ nos portadores de FM. Confirmamos escores significativamente superiores nos testes BASDAI ( $\mathrm{P}<0,001$ pelo teste $t$ de Student e $\mathrm{P}=0,002940$ pelo teste Wilcoxon $)$ e BASFI $(\mathrm{P}<0,001$ pelo teste $t$ de Student e $\mathrm{P}=0,001992$ pelo teste Wilcoxon). Além disso, comparamos os resultados do teste ASQoL e também verificamos escores superiores $(\mathrm{P}=0,0019$ pelo teste $t$ de Student e $\mathrm{P}=0,001884$ pelo teste Wilcoxon) entre os pacientes com FM. Heikkilä et al., ${ }^{23}$ ressaltam as correlações encontradas entre as duas doenças, apesar da predominância de cada uma delas em sexos e idades diferentes. Quando avaliadas laboratorialmente, apenas a EA apresenta características de doença inflamatória. Contudo, sob o aspecto clínico, ambas levam a redução da funcionalidade e capacidade laboral dos portadores de maneira muito semelhante. ${ }^{23-25}$ É importante considerar que EA e FM compartilham muitos sintomas. O diagnóstico de EA é baseado na presença de dor vertebral crônica associada à rigidez matinal, que por sua vez são queixas frequentes na FM. ${ }^{18}$ As queixas de ansiedade, depressão, fadiga e distúrbios do sono são significativas na FM e da mesma forma também são muito reportadas por pacientes com EA, problemas estes fortemente associados com a questão da dor. ${ }^{26-28}$

Atualmente, a indicação de uso de biológicos na EA é baseada no índice de atividade, dentre outros. ${ }^{22}$ Baseados em nossos resultados, sugerimos que antes da indicação do uso de biológicos, pacientes com EA sejam avaliados para a possibilidade da presença concomitante de FM e aqueles que apresentarem critérios positivos sejam adequadamente tratados e reavaliados.

Uma das limitações para análise de nosso estudo é o fato de não termos lançado mão de uma população controle. Contudo, mesmo com um delineamento não controlado tratase da maior casuística de pesquisa de FM entre pacientes com EA publicada até o momento. Verificamos uma prevalência de $15 \%$ de FM na EA, sendo a concomitância distribuída de forma semelhante entre homens e mulheres. Além disso, os dados apontaram para o fato de que a presença concomitante da FM pode agravar os sintomas de atividade da doença, o impacto funcional e o prejuízo na qualidade de vida dos pacientes com EA. 


\section{REFERÊNCIAS}

\section{REFERENCES}

1. Sampaio-Barros P, Azevedo VF, Bonfiglioli R, Campos WR, Carneiro SCS, Carvalho MAP et al. Consenso Brasileiro de Espondiloartropatias: espondilite anquilosante e artrite psoriásica diagnóstico e tratamento - Primeira revisão. Rev Bras Reumatol 2007; 47(4):233-42.

2. Dougados M. Diagnostic features of ankylosing spondylitis. Br J Rheumatol 1995; 34:301-5.

3. Van Der Linden S, Van Der Heijde D. Ankylosing spondylitis: clinical features. Rheum Dis Clin North Am 1998; 24(4):663-76.

4. Reveille JD, Ball EJ, Khan MA. HLA-B27 and genetic predisposing factors in spondyloarthropathies. Curr Opin Rheumatol 2001; 13(4):265-72.

5. Rudwaleit M, Metter A. Inflammatory back pain in ankylosing spondylitis: a reassessment of the clinical history for application as classification and diagnostic criteria. Arthritis Rheum 2006; 54(9):569-78.

6. Van der Linden S, Valkenburg HA, Cats A. Evaluation of diagnostic criteria for ankylosing spondylitis: a proposal for modification of the New York criteria. Arthritis Rheum 1984; 27(4):361-8.

7. Garrett S, Jenkinson T, Kennedy LG, Whitelock H, Gaisford P, Calin A. A new approach to defining disease status in ankylosing spondylitis: the Bath Ankyosing Spondylitis Disease Activity Index. J Rheumatol 1994; 21(12):286-91.

8. Torres T, Ciconelli R. Instrumentos de Avaliação em Espondilite Anquilosante. Rev Bras Reumatol 2003; 46(1):52-9. 
9. Calin A, Garrett S, Whitelock H, Kennedy LG, O'Hea J, Mallorie $\mathrm{P}$ et al. A new approach to defining functional ability in ankylosing spondylitis: the development of the Bath Ankylosing Spondylitis Functional Index. J Rheumatol 1994; 21(12):2281-5.

10. Heikkilä S, Viitanen JV, Kautianen H, Kauppi M. Evaluation of the finnish versions of the functional indices BASFI and DFI in spondylarthropathy. Clin Rheumatol 2000; 19(6):464-9.

11. Udrea G, Ciobanu C, Mihai C, Stoica V, Suteanu S, Van Der Heijde D et al. Evaluation of Romanian version of the Bath Ankylosing Spondylitis functional index (BASFI) in patients with spondylarthropathies. Romanian Journal of Internal Medicine 2004; 42(11):199-209.

12. Doward L, Spoorenberg A, Cook S, Whalley D, Helliwell P, Kay L et al. Development of the ASQoL: a quality of life instrument specific to ankylosing spondylitis. Ann Rheum Dis 2003; 62(1):20-6.

13. Projeto Diretrizes - Sociedade Brasileira de Reumatologia. Fibromialgia. Acesso em: 20 de abril de 2010.

14. Wolfe F, Smythe HA, Yunus MB, Bennett RM, Bombardier C, Goldenberg DL et al. The American College of Rheumatology 1990 Criteria for the Classification of Fibromyalgia. Report of the Multicenter Criteria Committee. Arthritis Rheum 1990; 33(2):160-72.

15. Skare T. Reumatologia: princípios e prática. 2 Ed. Rio de Janeiro: Guanabara Koogan, 2007, pp. 335.

16. Buskila D, Langevitz P, Gladman DD, Urowitz S, Smythe HA. Patients with rheumatoid arthritis are tenderer than those with psoriatic arthritis. J Rheumatol 1992; 19(7):1115-19.

17. Buskila D, Odes LR, Neumann L, Odes HS. Fibromyalgia in Inflammatory bowel disease. J Rheumatol 1999; 26(5):1167-71.

18. Wolfe F, Smythe HA, Yunus MB, Bennett RM, Bombardier C, Goldenberg DL et al. The American College of Rheumatology 1990, Criteria for the Classification of Fibromyalgia. Report of the Multicenter Criteria Committee. Arthritis Rheum 1990; 33(2):160-72.
19. Aloush V, Ablin J, Reitblat T, Caspi D, Elkayam O. Fibromyalgia in women with ankylosing spondylitis. Rheumatol Intern 2007; 27(9):865-8.

20. Brewerton DA, Caffrey M, Hart FD, James DCO, Nicholls A, Sturrock RD. Ankylosing spondylitis and HLA-B27. Lancet 1973; 1:904-7.

21. Van Der Linden SM, Valkenburg HA, De Jongh BM, Cats A. The risk of developing ankylosing spondylitis in HLA-B27 positive individuals: a comparison of relatives of spondylitis patients with the general population. Arthritis Rheum 1984; 27(3):241-9.

22. Sieper J, Braun J, Rudwaleit M, Boonen A, Zink A. Ankylosing spondylitis: an overview. Ann Rheum Dis 2002; 61(3)27:241-9.

23. Heikkilä S, Ronni S, Kautiainen HJ, Kauppi MJ. Functional impairment in spondyloarthropathy and fibromyalgia. J Rheumatol 2001; 29(7):1415-19.

24. Turan Y, Duruöz MT, Bal S, Guvenc A, Cerrahoglu L, Gurgan A. Assessment of fatigue in patients with ankylosing spondylitis. Rheumatol Intern 2007; 27(9):847-52.

25. Boonen A, Van Den Heuvel R, Van Tubergen A, Goossens M, Severens J, Van Der Heijde D et al. Large differences in cost of illness and wellbeing between patients with fibromyalgia, chronic low back pain, or ankylosing spondylitis. Ann Rheum Dis 2005; 64(3):396-402.

26. Dernis-Labous E, Messow M, Dougados M. Assessment of fatigue in the management of patients with ankylosing spondylitis. Rheumatology 2003; 42(12):1523-8.

27. Barlow JH, Macey SJ, Struthers GR. Gender, depression, and ankylosing spondylitis. Arthritis Care Res 1993; 6(1):45-51.

28. Hultgren S, Broman JE, Gudbjornsson B, Hetta J, Lindqvist U. Sleep disturbances in outpatients with ankylosing spondylitis questionnaire study with gender implications. Scand J Rheumatol 2000; 29(6):365-9. 